\title{
Composition algebras and the two faces of $G_{2}$
}

\author{
Luis J. BOYA \\ Departamento de Física Teórica, \\ Universidad de Zaragoza, \\ E-50009 Zaragoza, Spain. \\ luisjo@unizar.es \\ Rutwig Campoamor-Stursberg \\ Instituto de Matemática Interdisciplinar and \\ Universidad Complutense de Madrid \\ 3, plaza de Ciencias, E-28040 Madrid, Spain \\ rutwig@pdi.ucm.es
}

November 17, 2009

\begin{abstract}
We consider composition and division algebras over the real numbers: We note two rôles for the group $G_{2}$ : as automorphism group of the octonions and as the isotropy group of a generic 3 -form in 7 dimensions. We show why they are equivalent, by means of a regular metric. We express in some diagrams the relation between some pertinent groups, most of them related to the octonions. Some applications to physics are also discussed.
\end{abstract}

\section{Introduction}

The first exceptional Lie group $G_{2}$ (dimension 14, rank 2) was first discovered, as its complex Lie algebra, in 1887 by W. Killing, in his (redundant) list of all complex simple Lie algebras; the list was fixed by É. Cartan in his Paris Thesis (1894). $G_{2}$ is the only Lie group which has a Dynkin diagram with a triple bond, $\bullet \equiv \circ$ : the two smallest representations $(\neq \mathrm{Id})$ are of dimensions 7 and 14. In physics the group $G_{2}$ appears in several context, as in 7-dim compactifications from M-theory.

On the other hand, the division algebra of the octonions was first written by J. Graves in Christmas, 1843 (letter to W. Hamilton, who soon realized they were non-associative); however, the eight-squares sum, related also to octonions (as the four- squares sum (Euler, 1738) was related to the quaternions), had been found earlier by C. Degen (1818); in 1845 A. Cayley also (re)discovered the octonions in relation to his work on hyperelliptic functions; so the name Cayley numbers, octaves and octonians are also used in the literature for the octonions.

É. Cartan was the first to consider the group $G_{2}$ (in its real compact form) as the automorphism group of the octonion algebra (1914; it is quoted even in an earlier article of his in 1908); he also showed there exists another, noncompact real form, that today we know it is the automorphism group of the split octonions. But already in 1900, F. Engel (who wrote with S. Lie the monumental 3-volume book on Transformation Groups [3]) established (the complex form of) $G_{2}$ as the isotropy group of a generic 3 -form in 7 -dimensional complex space; 
his disciple W. Reichel worked out the details of this description of $G_{2}$, including the two real forms, in 1907. In modern times (ca. 1984) this 3-form has been much used by Bryant [12] in relation to manifolds of $G_{2}$ holonomy. All this historical record is narrated in detail in [1] and [2], see also [4, 5].

This paper is devoted to the relation between composition and division algebras and the two approaches for $G_{2}$; indeed, the two conceptions $\left(G_{2}=\right.$ Aut (Octonions) and $G_{2}=$ isotropy group of a generic 3-form in 7 dimensions) have been already noticed as equivalent by [2, 1] and others, but it seems to us rather obscure the stated relations (one exception is [14]). Specifically, we shall construct the octonions starting with the regular 3 -form $\phi$, wherefrom the dual role of $G_{2}$ will be evident: the 3 -form determines a regular metric $\beta$, which converts the form $\phi$, as $(0,3)$-tensor, in a $(1,2)$-tensor: that is, an algebra $\gamma=\beta(\phi)$, which turns out to be, of course, the octonion algebra: the group $G_{2}$ becomes automatically the automorphism group of that algebra. In the work of Bonan [6], a reverse result seems to be stated: starting from Cartan's description of compact $G_{2}$ as the automorphism group of the octonions, he saw how to derive from its multiplicative law an invariant 3-form. Before this we shall elaborate on composition and division algebras and then also extend some remarks of [2] on the relations of Spin(8), Spin(7) (both have a 8-dim real representation) and other smaller groups, including $G_{2}$, to the above work. Several physical applications will be also briefly mentioned, including some rôle for $S U(3)$ connected with octonions.

But first we need to recall some important fact about reals $\mathbb{R}$, complex $\mathbb{C}$, quaternions $\mathbb{H}$ and octonions $\mathbb{O}$, and their split forms (for the last three).

\section{Complex and quaternions as composition algebras}

If $\mathbb{F}$ is a field of numbers, an algebra $A=A(\mathbb{F})$ is a vector space over $\mathbb{F}$ with a linear map: $A \times A \rightarrow A$ distributive with respect to addition. We shall not assume commutativity nor associativity in the algebra. Typical examples are the complex numbers $\mathbb{C}$ over the reals $\mathbb{R}$, or the generic linear maps $\operatorname{End}(V)$ for $V$ an $n$-dim vector space over $\mathbb{F}$.

An algebra is called composition algebra, if there is a bilinear regular symmetric form $\beta: A \times A \rightarrow \mathbb{F}$ whose associated quadratic form $Q(a):=\beta(a, a)$ satisfies $Q(a b)=Q(a) Q(b)$; we shall usually take $\mathbb{F}=\mathbb{R}$ or $\mathbb{C}$. It is called division algebra if $a b=0$ implies either $a=0$ or $b=0$; it is called normed algebra if it has a norm as a vector space $N: A \rightarrow \mathbb{R}$ verifying $N(a \neq 0)>0$ and $N(a b)=N(a) N(b)$; if $A$ is division algebra, then $Q=Q(A)$ is definite, and $N$ is $+\sqrt{Q}$ : only division algebras can be normed, although sometimes the norm is defined as $N=+\sqrt{|Q|}$.

Let us briefly recall the composition algebras over the reals, $\mathbb{F}=\mathbb{R}$. An algebra on $\mathbb{R}^{2}$ is determined once $J^{2}$ is known, where $J:=\{0,1\}$ is a second unit vector, besides $1=\{1,0\}$. There are three cases [4]: if $J^{2}=0$, we have the degenerate complex numbers $\mathbb{C}^{0}$; we shall not consider them any further. If $J^{2}=-1$, we have the ordinary complex numbers $\mathbb{C}$, and if $J^{2}=+1$, we have the split complex numbers $\mathbb{C}^{\prime}$; for $\mathbb{C}$ and also for $\mathbb{C}^{\prime}$ we have the involutory automorphism $z=x+J y \mapsto \bar{z}=x-J y$, with $x, y$ reals. Then $Q(z):=\bar{z} z \in \mathbb{R}$ is a quadratic form, and as $Q\left(z z^{\prime}\right)=Q(z) Q\left(z^{\prime}\right)$, we have two possible composition algebras, for the complex numbers $\mathbb{C}$ and for the split complex $\mathbb{C}^{\prime}$. For $\mathbb{C}$, we have a field (=commutative division algebra), because $z^{-1}:=\bar{z} / Q(z), z \neq 0$, makes sense, whereas in $\mathbb{C}^{\prime}$, the split case, $Q(z)=0$ implies that $z$ lies in any place in the "light cone" $x= \pm y$. So both the complex and the split complex make up a composition algebra, but only the complex $\mathbb{C}$ are also a division algebra and it is a field, as it is commutative. For both $\mathbb{C}$ and $\mathbb{C}^{\prime}$ we have the two-squares identity, which are just 
the expression of the composition property $Q\left(z z^{\prime}\right)=Q(z) Q\left(z^{\prime}\right)$ : for $z=x+J y, z^{\prime}=x^{\prime}+J y^{\prime}$, $J^{2}= \pm 1$; known from antiquity, they are

$$
\begin{array}{lr}
\left(x^{2}+y^{2}\right)\left(x^{\prime 2}+y^{\prime 2}\right)=\left(x x^{\prime}-y y^{\prime}\right)^{2}+\left(x y^{\prime}+y x^{\prime}\right)^{2} & \text { from } \mathbb{C}\left(J^{2}=-1\right) \\
\left(x^{2}-y^{2}\right)\left(x^{\prime 2}-y^{\prime 2}\right)=\left(x x^{\prime}+y y^{\prime}\right)^{2}-\left(x y^{\prime}+y x^{\prime}\right)^{2} & \text { from } \mathbb{C}^{\prime}\left(J^{2}=+1\right)
\end{array}
$$

If $\mathbb{C}^{*}=\mathbb{C}-\{0\}$ is the multiplicative group of the field $\mathbb{C}$, we have topologically $\mathbb{C}^{*} \simeq \mathbb{S}^{1} \times \mathbb{R}^{+}$ (polar decomposition), where $\mathbb{S}^{1}$ are just the $Q(z)=1$ complex numbers and $\mathbb{R}^{+}$the positive reals. The automorphisms of both $\mathbb{C}$ and $\mathbb{C}^{\prime}$ as $\mathbb{R}$-algebras are just complex conjugation, Aut $(\mathbb{C})$ $=\operatorname{Aut}\left(\mathbb{C}^{\prime}\right)=\mathbb{Z}_{2}=O(1) \simeq \mathbb{S}^{0}\left(O(n)\right.$ is the $n$-dim real orthogonal group, $n \geq 1$, and $\mathbb{S}^{n}$ the $n$-sphere, $n \geq 0$ ); the fix point set is the real line (Recall the complex as a field has other (discontinuous) automorphisms).

The story is analogous in the next step, the (split) quaternions (Hamilton, 1843). One has now two units, $i$ and $j$, both of square \pm 1 ; then $k:=i j$ has to be another unit, so one has the vector space $\mathbb{R}^{4}$. For the division algebra case, $\mathbb{H}, i^{2}=j^{2}=k^{2}=-1$, the three $e: i, j$ and $k$ are anti-involutory $\left(e^{4}=1\right)$ and anticommuting, and then, if $q$ is a generic quaternion, $q=u+\boldsymbol{\sigma} \cdot \boldsymbol{x}$ $=$ real plus imaginary, where $\boldsymbol{\sigma}=\{i, j, k\}$ and $\mathbf{x}$ is a real 3-vector, the conjugation $q \mapsto \bar{q}$ is an (anti-)automorphism, and again $Q(q):=\bar{q} q \in \mathbb{R}$ is a quadratic form, definite, $Q(q)=u^{2}+\mathbf{x} \cdot \mathbf{x}$ : sum of real squares, satisfying $Q\left(q q^{\prime}\right)=Q(q) Q\left(q^{\prime}\right)$. The inverse is $q^{-1}=\bar{q} / Q(q), q \neq 0$, and one has the division algebra of the quaternions $\mathbb{H}$. Notice the conventional vector product in 3-dim is $\mathbf{x} \wedge \mathbf{y}=\operatorname{Im}(\mathbf{x y})=[\mathbf{x}, \mathbf{y}] / 2$, where $\mathbf{x}, \mathbf{y}$ are imaginary quaternions, and the scalar product verifies $\mathbf{x} \cdot \mathbf{y}=-\operatorname{Re}(\mathbf{x y})$. The pair $q=(u, \mathbf{x})$ was already called by Hamilton the scalar $(u)$ and the vector $(\mathbf{x})$ part of the quaternion; the "noncommutative field" of the quaternions is named at times a skew-field.

To get the split quaternions $\mathbb{H}^{\prime}$ one takes one of the units involutory, e.g. $i^{2}=+1$. Then everything works similarly as in the split complex case, with now $j^{2}=-1$ and $k^{2}=(i j)^{2}=+1$, and one still has a composition algebra; but the quadratic form is of $(++--)$ signature: $Q(q)=u^{2}-x^{2}+y^{2}-z^{2}$; (if the three units have square +1 , they do not anticommute, and there is no composition algebra!).

It is interesting to look at (anti)automorphisms of these algebras $\mathbb{H}$ and $\mathbb{H}^{\prime}$. For the (true) quaternions $\mathbb{H}$, it is easy to see that any rotation in the 3-space of unit imaginary $q$ 's is an automorphism, and viceversa, so $\operatorname{Aut}(\mathbb{H})=S O(3)$ : any frame $\epsilon(i, j, k=i j)$ is rotated in another one $\epsilon^{\prime}$ with the same orientation. Conjugation is an antiautomorphism, i.e. $\overline{\left(q q^{\prime}\right)}=\overline{q^{\prime}} \bar{q}$, so the group of autos and antiautos AntiAut $(\mathbb{H})$ is $O(3)$. There is also the group $\mathbb{Z}_{3}=A_{3}$ permuting cyclically the imaginary units $i, j$ and $k$, as it is an oriented frame. The $S O(3)$ automorphism group leaves the imaginary volume form (a 3-form) invariant; the quadratic form $Q(q)$ is invariant under $O(4)$, of course.

For the split quaternions $\mathbb{H}^{\prime}$ the automorphism group is the connected part of the orthogonal group for the $(++-)$-metric, $S O_{0}(2,1)$, and there is no cyclic symmetry. In the full split quaternions $\mathbb{H}^{\prime}$ the metric is of signature $(2,2)$, so the isometry group of $Q(q)$ is now $O(2,2)$. Unit quaternions make up $\mathbb{S}^{3}=S U(2)=S q(1)=\operatorname{Spin}(3)$ (the double and universal cover of $S O(3)$ ), and can be used efficiently to describe rotations in 3- and 4-space [7]. We also have the "polar" decomposition $\mathbb{H}^{*}=\mathbb{H}-\{0\} \simeq \mathbb{S}^{3} \times \mathbb{R}^{+}$, where $\mathbb{S}^{3} \simeq S U(2)$. We name $S q(n)$ the "unitary" $n \times n$ group, with quaternion entries.

It is remarkable that at this level, for the composition character only anticommutativity $\{i, j\}=0$ plays a role, whereas for the division property one needs also the anti-involutory condition, that is, $J^{2}=-1$ for the three units $i, j, k$. 
We omit the corresponding two four-squares identities (Euler) for $\mathbb{H}$ and $\mathbb{H}^{\prime}$, analogous to (2.1) and (2.2), for brevity.

The application of complex numbers in physics needs no apology; we only mention here that the very first formulations of Quantum Mechanics incorporate the $i$, both in Heisenbergs matrix mechanics (June, 1925) as in the more widespread Schrödinger wave equation (January, 1926).

Quaternions are used in the 3-dim vector calculus, as stated above, although generally without mentioning the origin in the quaternion algebra. Rotations in Quantum Mechanics again use the covering group, $S q(1)=\operatorname{Spin}(3)$, but the usual notation, as $S U(2)$, makes it easy to forget the true nature is really the unit quaternions. But quaternion product as a way to quickly compose 3-dim rotations are used in many devices, even in some electronic games...

\section{Octonions and split octonions}

If we now have three algebraically independent units $i, j, k$, one has to go to $\mathbb{R}^{8}$, with units $1 ; i, j, k ; i j, j k, k i ;(i j) k$. For the (true) octonions $\mathbb{O}$ the seven units anticommute and square to -1 . Define $o:=u+\boldsymbol{\rho} \cdot \xi,(8=1+7)$, where $u$ is real, $\xi$ a real 7 -vector, and $\boldsymbol{\rho}$ is a short for the seven imaginary units; define routinely the conjugate as $\bar{o}=u-\boldsymbol{\rho} \cdot \xi$, so $Q(o):=$ $\bar{o} o=u^{2}+\xi^{2} \geq 0$, and inverse as $o^{-1}=\bar{o} / Q(o), o \neq 0$. One sees at once that in order $\bar{o} o$ to be real you need alternativity: $i(j k)=-(i j) k$ en lieu of associativity: this is the peculiar property of the octonions (and what made Hamilton to reject them). Just here composition character implies alternativity (and a hint of Hurwitz theorem: you cannot proceed beyond $\operatorname{dim} 8$ with composition algebras), whereas the division condition would imply again antiinvolutory character of all seven imaginary units. Then $o=\operatorname{Re} o+\operatorname{Im} o(8=1+7)$, with norm $N(o)=|o|=+\sqrt{\bar{o} o}$; in this division algebra case the $Q$ is definite. Another way to obtain alternativity is to insist that two of the three units $(i, j, k)$ generate a quaternion algebra. The precise definition of alternativity is this: an algebra $A$ is alternative, if the alternator $[a, b, c]:=(a b) c-a(b c)$ is fully antisymmetric in the three arguments: any associative algebra is alternative, of course, as $[a, b, c]=A-A=0$. The polar decomposition is again $\mathbb{O}^{*} \simeq \mathbb{S}^{7} \times \mathbb{R}^{+}$, on the understanding that $\mathbb{S}^{7}$ is not a group (it has a structure called a loop). We recall that the four spheres $\mathbb{S}^{0}, \mathbb{S}^{1}, \mathbb{S}^{3}$ and $\mathbb{S}^{7}$ are the only parallelizable ones, and clearly described the $Q(x)=1$ numbers for $x$ in $\mathbb{R}, \mathbb{C}, \mathbb{H}$ and $\mathbb{O}$ respectively.

For the split octonions $\mathbb{O}^{\prime}$ we can take e.g. $\left(e_{1}=i\right)^{2}=+1$, and then $\left(e_{1} e_{2}\right)=e_{4},\left(e_{3} e_{1}\right)=e_{6}$ and $e_{7}$ are also involutive, so the 7-dim metric has signature $(+--+-++)$, and $Q=\bar{o} o$ has $(4,4)$ signature; of course, one still needs alternativity to guarantee $Q\left(o o^{\prime}\right)=Q(o) Q\left(o^{\prime}\right)$.

The situation is thus nearly identical for the three division algebras $\mathbb{C}, \mathbb{H}$ and $\mathbb{O}$ : the new units are anti-involutory, and have to anticommute in this, the division algebra case; $\mathbb{C}$ is commutative and associative, $\mathbb{H}$ is associative but not commutative, and $\mathbb{O}$ is neither. Anticommutativity restricts non-commutativity for quaternions, and alternativity substitutes non associativity for octonions; as there are no more properties to sacrify, there are neither composition nor divison algebras in higher dimensions, although there are some rings, called e.g. sedenions (dim 16) for the next case, of four algebraically independent units: if one proceeds to these sedenions, with four units $i, j, k, l$, and $(1+4+6+4+1)=16$ dimensions, the possible algebras are neither composition nor division (Hurwitz, 1896; Zorn, 1930). The process, which is a variant of the Cayley-Dickson method [1], continues...

A nice way to represent the octonions is the Fano plane $\mathbb{F}_{2} \mathbb{P}^{2}$ (see e.g. [1]), the projective plane over the (Galois) field of two elements $\mathbb{F}_{2}$ : it draws the $7=p^{2}+p+1$ (for $p=2$ ) 
imaginary units in a triangle (three vertices, three mid-lines and the center). Recall the order of the (projective) symmetry group $P G L_{3}(2)=P S L_{2}(7)$ is $168=\left(2^{3}-1\right)\left(2^{3}-2\right)\left(2^{3}-4\right)=$ $\left(7^{2}-1\right)\left(7^{2}-7\right) / 2$, and it is the second smallest non-Abelian simple group, the first one being $A_{5}=P S L_{2}(5)$, of order $60=5 ! / 2$.

What about octonion automorphisms? The possible group Aut $(\mathbb{O})$ has to lie inside $S O(7)$, with $\operatorname{dim} 21$, because there is norm and orientation to preserve; let us just call $\operatorname{Aut}(\mathbb{O}):=G_{2}$; it has to be smaller than $S O(7)$, because not any 7-frame goes into any other: both Baez [1] and Rosenfeld [4] make easy the case for $G_{2}$ to have dimension 14; taking $i=e_{1}$ in the 6-dim sphere of unit imaginary octonions, $j=e_{2}$ has to lie in the orthogonal equator $\left(\mathbb{S}^{5}\right)$, and $k=e_{3}$ in the 3 -dim space orthogonal to $(i, k$ and $i j$ ): so $\operatorname{dim} \operatorname{Aut}(\mathbb{O})=6+5+3=14$ (this shorter argument is also in ([7], p. 76)). Then, as $G_{2}$ acts transitively in the 6-sphere of unit imaginary octonions, the isotropy group $K \subset G_{2}$ has dimension $14-6=8$ : it turns out to be the $S U(3)$ group with the real irreducible 6 -dim representation (becoming $\overline{3}+3$ over the complex): $S U(3)$ leaves the $\mathbb{S}^{5}$ diameter of the previous 6 -sphere fixed, embeddable in a $\mathbb{R}^{6}$ space; we have then $G_{2} / S U(3) \simeq \mathbb{S}^{6}$ as an homogeneous space (it turns out not to be a symmetric space), and also $S U(3) / S U(2)=\mathbb{S}^{5}$, as the exact sequence is $S U(2) \rightarrow S U(3) \rightarrow \mathbb{S}^{5} \subset \mathbb{R}^{6}$. See diagrams at the end of the paper, in Sect. 6.

What about a discrete group, in this octonionic case, which would play the role of the $\mathbb{Z}_{3}=A_{3}$ in the quaternion case? The seven imaginary units can be cyclically permuted, which gives a $\mathbb{Z}_{7}$ group, but this is not all: the Fano projective plane is also the projective line over $\mathbb{F}_{7}$; one has also triangular $2 \pi / 3$ rotations as symmetries, and they combine with $\mathbb{Z}_{7}$ to make up a non-Abelian group of order 21. The natural group acting on the Fano plane is $P G L_{2}(7)$, of order 336, while $\left|P S L_{2}(7)\right|=168$. Our discrete group is thus

$$
\mathbb{Z}_{7} \ltimes \mathbb{Z}_{3},
$$

where $\mathbb{Z}_{3} \subset \mathbb{Z}_{6}=\operatorname{Aut}\left(\mathbb{Z}_{7}\right)$ determines the semidirect product. This corresponds to the $A_{3}$ case for the quaternions, but here $\mathbb{Z}_{7} \ltimes \mathbb{Z}_{3} \subset A_{7}$; incidentally, this 21 order non-Abelian group is the only other, with the direct sum abelian group $\mathbb{Z}_{3} \oplus \mathbb{Z}_{7}$, of this order.

Finally unit imaginary octonions form $\mathbb{S}^{6}$, which admits a quasi-complex structure (BorelSerre) due to imaginary octonion multiplication: among the even spheres only $\mathbb{S}^{2}$ and $\mathbb{S}^{6}$ admit a (quasi-)complex structure, truly complex for $\mathbb{S}^{2}$.

Incidentally, it was Richard Feynman who first established a kind of 7-dimensional vector product, $(\boldsymbol{\xi} \wedge \boldsymbol{\eta})=[\boldsymbol{\xi}, \boldsymbol{\eta}] / 2=\operatorname{Im}(\boldsymbol{\xi} \boldsymbol{\eta})$, for $\boldsymbol{\xi}, \boldsymbol{\eta}$ imaginary octonions, generalizing the HamiltonGibbs vector calculus in three dimensions [9].

The automorphism group of the split octonions $\mathbb{O}^{\prime}$ is the noncompact real form of $G_{2}$, which lives inside $S O_{0}(4,3)$. The split octonion quadratic form $Q^{\prime}$ admits the $O(4,4)$ group as isometry, of course.

Modern high-energy physics uses many groups associated with the octonions, as $G_{2}$ (holonomy of compactifying from 11-dim space), $E_{8}$ as gauge group of the M-Theory still in 11-dim, not to speak of $E_{8}^{2}$, used in string theory, or $E_{6}$, which appears in Grand Unification Theories (GUTs).

\section{Some classes of tensors}

We start now a seemingly totally independent development. Consider $T_{q}^{p}$ (=tensors on a vector space, say $V$ over a field $\mathbb{F}$ ); take a particular one, $t \in T_{q}^{p}$; imagine the general linear group, with $g \in G L(V)$ acting on it in the natural way, write $g \cdot t=t^{\prime}$, and try to classify the tensors 
by equivalence classes (orbits) under the $G L$ action: as $\operatorname{dim} G L(V)=n^{2}$, the simple vectors $x$ (as $T_{0}^{1}$ tensors) are classified: the zero vector and the rest; the little group of the later orbit is the affine group $A_{n-1}$. Rank two covariant tensors $T_{2}^{0}$ ( $\Longleftrightarrow$ bilinear forms) split first into symmetric and antisymmetric parts, and there is also regular character (or not): any bilinear form $b \in T_{2}^{0}$ generates a linear map from $V$ to the dual space $V^{*}, b^{\prime}: V \rightarrow V^{*}$, and as they have the same dimension, the form $b$ is called regular if the map $b^{\prime}$ is an isomorphism (for good reasons, Hitchin [15] speaks of stable forms instead of regular). The isotropy groups for the regular case give rise to the orthogonal and symplectic groups in the known way. We recall:

If $Q$ is a regular quadratic form, the isotropy group $\{g, g \cdot Q=Q\}$ defines an orthogonal group $O(n)$; if $\omega$ is a regular 2 -form, the group is the symplectic one, $S p(n)$ (acting on even dimension $2 n$ space); if $\tau$ is a volume form (or $n$-form $\neq 0$ ), the isotropy group is the unimodular group, $S L(n)$ : so over any field, we have the three classical series of matrix groups of Cartan: $B-D, C$ and $A$; for some fields, e.g. over the reals, there is a further distinction by the Sylvester signature.

The natural question now arises: Is there any other orbits possible, tensors under $G L(V)$, besides the obvious $Q, \omega, \tau$ and with isotropy groups? If so, which (new) groups arise? For endomorphisms $\operatorname{End}(V) \simeq T_{1}^{1}$ the orbits under $G L(n)$ are the so-called "elementary divisors", which classify matrices; their little groups are easily identified, and are not very interesting for our purposes. The next possible case are $p$-forms [14], with dimension $\operatorname{dim} \bigwedge T_{p}^{0}\left(\mathbb{F}^{n}\right)=\left(\begin{array}{l}n \\ p\end{array}\right)$, but as $\left(\begin{array}{l}9 \\ 3\end{array}\right)=84>9^{2}=81$, we have potentially four cases only:

- 3-forms on $\mathbb{F}^{7} \quad\left(7^{2}=49>\left(\begin{array}{l}7 \\ 3\end{array}\right)=35\right)$.

- 3-forms on $\mathbb{F}^{8} \quad\left(8^{2}=64>\left(\begin{array}{l}8 \\ 3\end{array}\right)=56\right)$.

- (Self)-3-forms in $\mathbb{F}^{6} \quad\left(6^{2}=36>\left(\begin{array}{l}6 \\ 3\end{array}\right)=20>\frac{1}{2}\left(\begin{array}{l}6 \\ 3\end{array}\right)=10\right)$.

- Self-4-forms in $\mathbb{F}^{8}\left(8^{2}=64>\frac{1}{2}\left(\begin{array}{l}8 \\ 4\end{array}\right)=35\right.$.

As we have $\frac{1}{2}\left(\begin{array}{c}2 n \\ n\end{array}\right)=\left(\begin{array}{c}2 n-1 \\ n-1\end{array}\right)=\left(\begin{array}{c}2 n-1 \\ n\end{array}\right)$, we have $\operatorname{dim}\left(\right.$ self-3-forms in $\left.\mathbb{F}^{6}\right)=10=\operatorname{dim}(2$-forms in $\mathbb{F}^{5}$ ), but the 3 -forms themselves are very interesting, the isotropy group being $S L_{3}(\mathbb{C})^{2}$ for $\mathbb{F}=\mathbb{C}\left(6^{2}-2 \cdot 10=16\right)$ and $S L_{3}(\mathbb{C})$ for $\mathbb{F}=\mathbb{R},[14$. For $\mathbb{F}=\mathbb{C}$ the really interesting case is the generic 3 -forms in $\mathbb{C}^{7}$. So for a generic 3 -form $\phi$, we have $\operatorname{dim} \operatorname{Aut}(\phi)=49-35=14$, the same as the dimension of complex $G_{2}$ ! In fact, Bryant [12] bases his study of $G_{2}$ as invariance group of $\phi$. Engel and Reichel (see above) determined that there are two regular (stable) real forms, with isotropy the compact and noncompact forms of real $G_{2}$.

Is there any sensible isotropy group for a generic 3 -form in $\mathbb{R}^{8}$ ? It will be of dimension $8^{2}-\left(\begin{array}{l}8 \\ 3\end{array}\right)=64-56=8$, and in the compact case the candidate would be $S U(3) / \mathbb{Z}_{3}=\mathbb{P U}(3)$, which has a nice 8-dim real representation, in fact the adjoint of $S U(3)$; but we do not consider this case anymore (see again [14, 15]), except the trivial remark that then the $S U(3)$ group would appear a second time in relation to $\mathbb{R}^{8}$ (and the octonions), with possible applications in physics: both flavour and colour physics use the group $S U(3)$ consistently.

The (anti-)self-dual 4-forms are classes under $S L_{8}(\mathbb{R})$ and have also dimension 35; they are called Cayley forms. They are not generic; the pertinent group happens to be $\operatorname{Spin}(7)$, which has also a single real 8-dim representation; a nice discussion is in ([8], p. 255), as Spin(7), together with $G_{2}$, are the two exceptional holonomy groups. In a precise way, which we do not elaborate, the 3 -form in $\mathbb{F}^{7}$ comes really from the self and antiselfdual forms in $\mathbb{F}^{8}$. 
The proof that there are no more left over cases is just numerical: $n^{2}=\operatorname{dim} G L_{n}(\mathbb{F})$ is less than any other generic tensor; the reader can be convinced by himself that generic tensors of any other rank would be equivalent to the above ones or have no room for isotropy groups.

\section{The 3-form in dimension 7 and the octonions}

Starting with a generic 3 -form $\phi$ in $\mathbb{F}^{7}$, if there is such a thing, the isotropy group, of dimension 14 , will be a certain group $G:=G(3 ; 7)$. One suspects, of course, that for $\mathbb{F}=\mathbb{R}, G(3 ; 7)=$ Aut $(\mathbb{O})$; how does one prove this? We shall only indicate the idea of the proof. Start by the 3 -form in $\mathbb{F}^{7}$ (for $\mathbb{F}=\mathbb{R}, \mathbb{C}$ ) and try to recover the octonions, with the group $G_{2}$ playing the dual role: it is the isotropy group of a "generic" 3 -form in $\mathbb{F}^{7}$ and at the same time the automorphism group of the (constructed) octonion algebra. The idea of the proof goes along the following steps:

1. There is also a sense of regularity in the 3-form: in fact, a such form $\phi$, generates an special bilinear symmetric form $\beta$ (found already by Engel, see [2, 14]): for $\mathbf{x}, \mathbf{y}$ vectors in $\mathbb{F}^{7}$, if $\phi(\mathbf{x})$ is the 2 -form contraction, $\left.\phi(\mathbf{x})=\mathbf{x}\right\lrcorner \phi$,

$$
\beta(\mathbf{x}, \mathbf{y}):=\phi(\mathbf{x}) \wedge \phi(\mathbf{y}) \wedge \phi, \quad(\text { i.e., a } 7-\text { form })
$$

2. Call $\phi$ regular if $\beta(\phi)$ is regular (non-degenerate). The isotropy group of this special 7 dimensional metric is $S O(7, \mathbb{F})$, and one recovers the natural inclusion $G(3 ; 7) \subset S O(7, \mathbb{F})$. Engel proved that, in the three cases $(\mathbb{F}=\mathbb{C}$ and the two $\mathbb{F}=\mathbb{R})$, the $\beta$ form is nondegenerate for a generic (=lying in an open set) 3 -form $\phi$.

3. The $S O$ group lies inside $S L(\mathbb{F})$, hence there is a volume element; therefore, there is the Hodge duality operator ${ }^{*}$, and there is also an invariant 4 -form $\psi={ }^{*} \phi$, as $\phi \wedge^{*} \phi=\tau$ is the volume element. Recall $\operatorname{dim} 3$-forms $=\operatorname{dim} 4$-forms for $n=7$.

4. With the regular metric $\beta$ and the 3 -form $\phi$, one gets an algebra! This is because an algebra is a (particular) $T_{2}^{1}$ tensor, as $x y=z$ means precisely this; the metric being regular, $\exists \beta^{-1}$, and one flips an index, passing from $\phi$ a $\wedge T_{3}^{0}$ tensor to a $T_{2}^{1}$ one; write $\beta^{1}(\phi)=\gamma$. Hence, in $\mathbb{F}^{7}$ one has an algebra, with $\gamma(\mathbf{x}, \mathbf{y})=\mathbf{z}$. It is antisymmetric, $\gamma\left(e_{1}, e_{2}\right)=e_{1} e_{2}=-e_{2} e_{1}$ (for a basis $e_{1}, \ldots, e_{7}$ ), because so is $\phi$ in two indices, and it is alternative, because $\phi$ is fully antisymmetric! See 8) below.

5. One has reproduced the "vector" product of imaginary octonions!, and so you can reconstruct the Fano plane for imaginary octonionic multiplication.

6. Adding now the unit 1 , so that $e_{i}^{2}$ is not zero but -1 (or +1 , see below), we have reconstructed the octonion composition and division algebras in the case $\mathbb{F}=\mathbb{R}$ ! And of course, now it is clear that the $G_{2}$ group has two faces: it is either the isotropy group of a 3-form, or automatically the Aut group of the algebra: this is the looked-for Two Faces of $G_{2}$. One shows also that $G_{2}$ is compact for the octonion division case (OO), and a noncompact form, lying in $S O_{0}(4,3)$, for the split case, where some of the units square to +1 . Engel and Reichel proved [2] that there is a unique generic class of generic 3-forms for $\mathbb{F}=\mathbb{C}$, and two for $\mathbb{F}=\mathbb{R}$.

7. Comparing this with the (true) quaternions $\mathbb{H}$, there the 3 -form is the volume form, with $S L_{3}(\mathbb{R})$ as isotropy group; but the metric is put by hand, so the group becomes $S L_{3} \cap O(3)=S O(3)$; and indeed $\operatorname{Aut}(\mathbb{H})=S O(3)$. 
8. For the concrete form of the 3-form one mimics the octonion product: if $\left\{e_{i}\right\}$ is a basis in, say, $\mathbb{R}^{7}$, with $\omega_{i}$ the dual basis, $\omega_{i}\left(e_{j}\right)=\delta_{i j}$, the generic 3 - form can be defined [12, 8 ] as

$$
\phi=(124)+(157)+(163)+(235)+(276)+(374)+(465),
$$

where (124) means $\omega_{1} \wedge \omega_{2} \wedge \omega_{4}$ etc. Passing through the metric $\beta$ and to the algebra $\gamma$, one should reproduce the octonion product of Sect. 3, to wit

$$
e_{1} e_{2}=e_{4}, e_{2} e_{3}=e_{5}, \ldots
$$

9. The argument above is oversimplified; for example, in the same way that a bilinear form over the reals might have a signature (Sylvester), it turns out that there are two cases of generic 3 -forms in $\mathbb{R}^{7}$ (Engel, Reichel). So one goes to the octonions and to the split octonions, as the isotropy group of these forms is compact and noncompact respectively.

10. Notice the difference between the two cases (corresponding to the true and split octonions) comes from adding the units, $e_{i}^{2}$, and does not depend much on the 7-dim structure (see 6 above).

\section{$6 \quad$ Spin groups}

We conclude by establishing the following relations between the several groups appearing in our study, inspired in ([8], p. 256); we first recall the coincidences among the small dimension spin groups (see e.g. [10]):

$$
\begin{array}{r}
\operatorname{Spin}(1)=O(1) ; \operatorname{Spin}(2)=S O(2)=U(1) ; \operatorname{Spin}(3)=S U(2)=S q(1) \\
\quad \operatorname{Spin}(4)=\operatorname{Spin}(3)^{2} ; \operatorname{Spin}(5)=S q(2) ; \operatorname{Spin}(6)=S U(4)
\end{array}
$$

We consider now the five groups with an irreducible representation of real dimension 8 , namely $S O(8)$, Spin(8), $S U(4), S q(2)$ and $\operatorname{Spin}(7)$; they are clearly related to $\mathbb{R}, \mathbb{C}, \mathbb{H}$ and $\mathbb{O}$; they also act transitively on the 7 -sphere $\mathbb{S}^{7}$. We shall consider three diagrams to illustrate these relations. Figure 1. shows the relation of the quaternions and the complex numbers, Figure 2. that of the octonions and the complex numbers, while considering the reals and the octonions yields the following diagram of Figure 3.:

Figure 1: $\mathbb{H}$ and $\mathbb{C}$

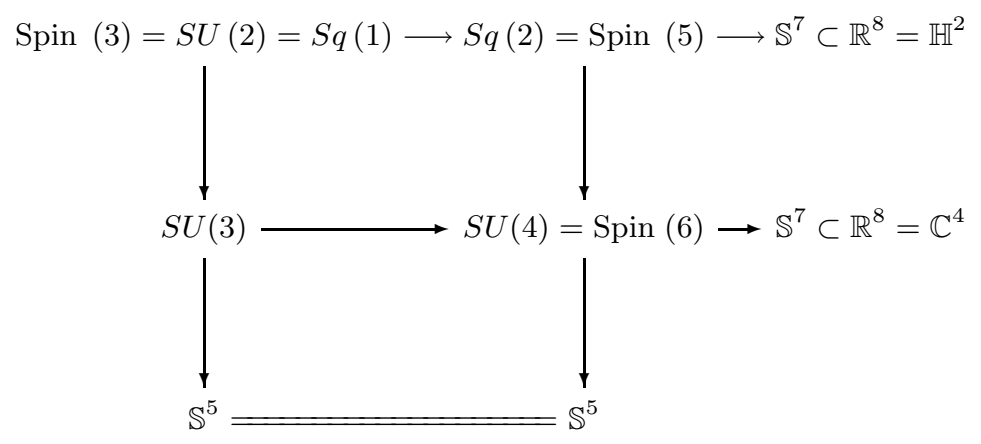


Figure 2: $\mathbb{O}$ and $\mathbb{C}$

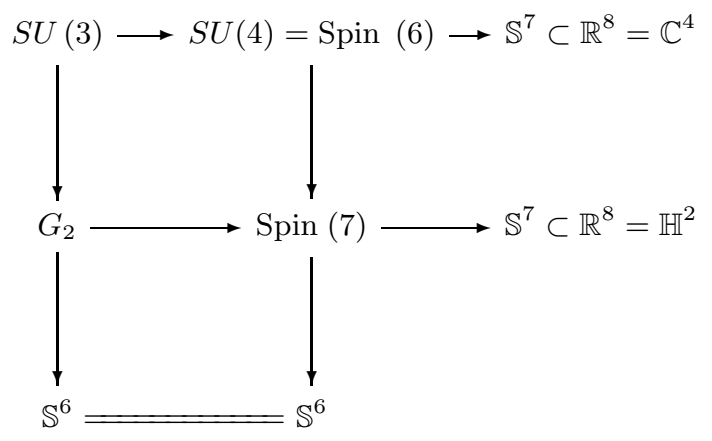

Figure 3: $\mathbb{R}$ and $\mathbb{O}$

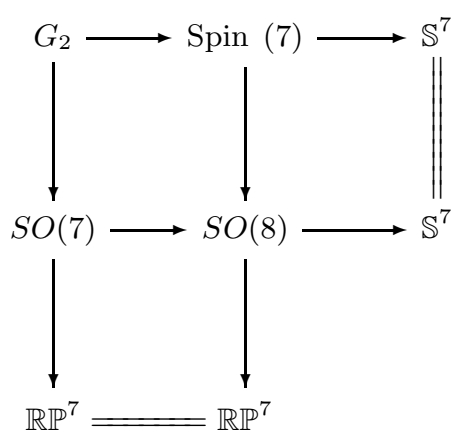

Only the first two columns require an explanation: as obviously $\operatorname{Spin}(8) / \operatorname{Spin}(7)=\mathbb{S}^{7}$, and $\operatorname{Spin}(8)$ covers $S O(8)$ twice, we have $S O(8) / \operatorname{Spin}(7)=$ the real projective space $\mathbb{R} P^{7} \simeq \mathbb{S}^{7} / \mathbb{Z}_{2}$.

The relation between spin groups and division algebras, in particular the octonion algebras, is noteworthy; we elaborate this in [11]. Lastly, a remark on Spin(8), which exhibits a wonderful triality: the extension of $O(8)$ by the three 8-dim representations (permuted by triality) gives rise to $F_{4}$, the second exceptional group, $\left(\left(\begin{array}{l}8 \\ 2\end{array}\right)+3 \cdot 8=52=\operatorname{dim} F_{4}\right)$, whereas the quotient of $O(8)$ by the same triality automorphism group generates $G_{2}$ (again!): the triple bond in the Dynkin diagram for $G_{2}$ (see Sect. 1) comes really from triality in $O(8) \simeq D_{4}$ ! [13].

The center of $\operatorname{Spin}(8)$, as any $\operatorname{Spin}(4 n)$ group, is $V=\mathbb{Z}_{2} \oplus \mathbb{Z}_{2}$ : and we know that $\operatorname{Aut}(V)$ is the symmetric group $S_{3}$; in all cases except $\operatorname{Spin}(8)$ (and $\mathrm{PO}(8)=\operatorname{Spin}(8) / V$ ) the outer automorphisms of the group shrinks to $\mathbb{Z}_{2}$ (which e.g. permutes the two chiral representations), but in $\operatorname{Spin}(8)$ the $S_{3}$ group of autos of the center lifts to a $S_{3}$ group of autos (triality!) of the full group: the deep reason of this is the "loop" multiplicative character of the seven sphere of unit octonions, which appears twice in $\operatorname{Spin}(8)$ : the sphere homology product of $\operatorname{Spin}(8)$ is $\mathbb{S}^{3} \odot \mathbb{S}^{7} \odot \mathbb{S}^{7} \odot \mathbb{S}^{11}$.

This triality is at the base of some supersymmetric field theories in physics, in particular $\mathcal{N}=1$ Susy Yang-Mills in $10=(1,1)+(8,0)$ dimensions, as well as the string theory IIA, with $\mathcal{N}=2$ Supersymmetries, involving the vector and the two spinor representations for the light cone group $O(8)$ as dictated by triality; see [16]. So in conclusion, as regards to physics, both the groups $S U(3) \subset G_{2}$ and triality (as enhancement of duality), which is connected with octonions, seem to be related, if not unavoidable, in modern physical theories. 
Even the group $F_{4}$ does appear in an unexpected place in physics: namely, in M-Theory or rather in the low-energy particle content. P. Ramond as shown [17] that the particles $h$ (graviton, $\operatorname{dim} 44), \Psi$ (gravitino, $\operatorname{dim} 128)$ and $C$ (3-form, $\operatorname{dim} 84$ ) are related to the Moufang octonionic plane, $\mathbb{O P}^{2}$, which as symmetric space is $F_{4} / \operatorname{Spin}(9)$ : the three represenations are induced from the Id representation of $F_{4}$, where the triplet is related to the Euler number, as $\chi\left(\mathbb{O P}^{2}\right)=3$. For further developments and extensions to F-Theory, see [18].

\section{Acknowledgements}

The first author (LJB) acknowledges partial financial support by the CICYT grant FPA20060235. The second author (RCS) acknowledges support by the research grant GR58/4120818920920 of the UCM-BSCH.

\section{References}

[1] J. C. Baez, The octonions, Bull. Am. Math. Soc. 39 (2001), 145-205. Errata, Ibid. 42 (2005), 213

[2] I. Agricola, Old and New on the Exceptional Group $G_{2}$, Notices A.M.S. 55 (2008), 922-929

[3] S. Lie and F. Engel, Theorie der Transformationsgruppen I, II und III (Leipzig, 1888).

[4] R. Rosenfeld, Geometry of Lie Groups, (Kluwer Academic, Amsterdam, 1997).

[5] R. Rosenfeld, A History of Non-Euclidean Geometry, (Springer, Berlin, 1988).

[6] E. Bonan, Sur les variétés riemanniennes à groupe d'holonomie $G_{2}$ ou $\operatorname{Spin}(7)$, C.R. Acad. Sc. Paris 262 (1966), 127-129.

[7] J. H. Conway and D. A. Smith, On Quaternions and Octonions (A.K. Peters, Natick, MA, 2003).

[8] D. Joyce, Compact Manifolds with Special Holonomy (Oxford U.P., Oxford, 2000).

[9] Unpublished: see F. J. Dyson, Feynman's proof of Maxwell equations, Am. J. Phys. 58 (1990), 209-211; read the story in Z. Silagadze, Feynmans derivation of Maxwell equations and extra dimensions, Ann. Fond. L. de Broglie 27 (2002), 241-255.

[10] F. R. Harvey, Spinors and Calibrations (Academic Press, New York, 1990).

[11] L. J. Boya, Spinors and octonions, arXiv:math-ph/0409077 (A. Galindo Festschrift, 2004).

[12] R. Bryant, Metrics with holonomy $G_{2}$ or Spin(7), in Lecture Notes in Mathematics Vol. 1111 (Springer, New York, 1984).

[13] J. F. Adams, Spin(8), triality, $F_{4}$ and all that, Selecta 2 (1981), 243-253.

[14] N. Hitchin, The geometry of three-forms in six and seven dimensions, J. Diff. Geom. 55 (2000), 547-576. arXiv:math.DG/0010054.

[15] N. Hitchin, Stable forms and special metric, arXiv:math/0107101 [math.DG].

[16] J. A. Harvey, Magnetic Monopoles, Duality and Supersymmetry, arXiv: hep-th 9603086. 
[17] T. Pengpan and P. Ramond, M(ysterious) patterns in SO(9), Phys. Rep. 315 (1999), $137-152$.

[18] L. J. Boya, Arguments for F-Theory, Mod. Phys. Lett. A 21 (2006), 287-304. 